\title{
LA-UR-18-28709
}

Approved for public release; distribution is unlimited.

Title: $\quad$ GRID MODERNIZATION INITIATIVE PEER REVIEW Extreme Event Modeling 1.4.17

Author(s): $\quad$ Bent, Russell Whitford

Intended for: $\quad$ GMLC Peer Review

Issued:

2018-09-12 
Disclaimer:

Los Alamos National Laboratory, an affirmative action/equal opportunity employer, is operated by the Los Alamos National Security, LLC for the National Nuclear Security Administration of the U.S. Department of Energy under contract DE-AC52-06NA25396. By approving this article, the publisher recognizes that the U.S. Government retains nonexclusive, royalty-free license to publish or reproduce the published form of this contribution, or to allow others to do so, for U.S. Government purposes. Los Alamos National Laboratory requests that the publisher identify this article as work performed under the auspices of the U.S. Department of Energy. Los Alamos National Laboratory strongly supports academic freedom and a researcher's right to publish; as an institution, however, the Laboratory does not endorse the viewpoint of a publication or guarantee its technical correctness. 


\section{GRID MODERNIZATION INITIATIVE PEER REVIEW Extreme Event Modeling 1.4.17}

\section{RUSSELL BENT}

September 4-7, 2018

Sheraton Pentagon City Hotel - Arlington, VA 


\section{Extreme Event Modeling 1.4.17}

\section{Natural and man-made extreme events pose threats}
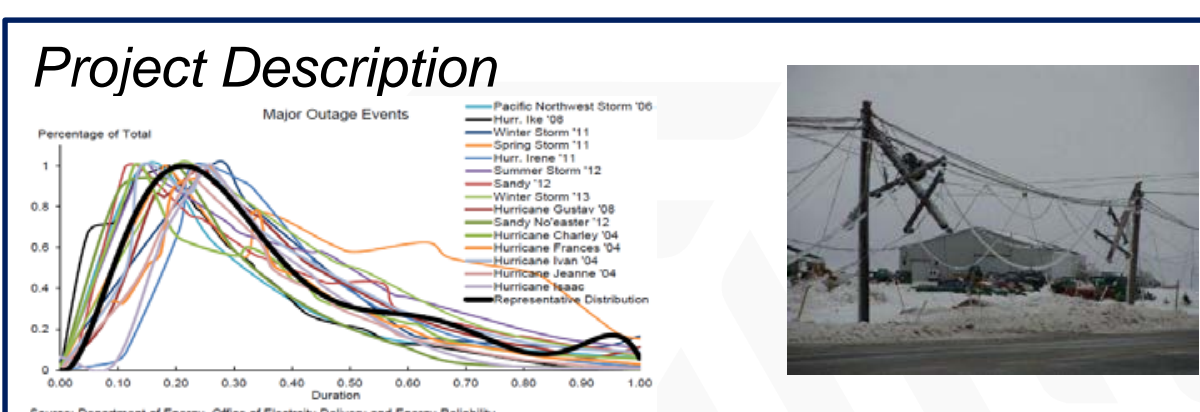

- Cascading and N-k modeling have large gaps

- Inadequate modeling

- Reliability standards (NERC Standard

TPL-001-4) challenging to meet with existing methods

- Computational efficiency

- Considerable speed up required for near term operations planning

- $\quad \mathrm{N}$-k contingency analysis

- $\quad$ Existing $\mathrm{k}=3$ analysis misses large-scale adversary attacks

- Neglects high likelihood failures

Value Proposition

$\checkmark \quad$ Identify extreme event risk prior to event occurrence

\section{Project Objectives}

$\checkmark$ Cascading tools that are 500x faster than existing packages

$\checkmark$ Identify the worst k contingencies twice as fast

$\checkmark$ Demonstration on a large-scale system

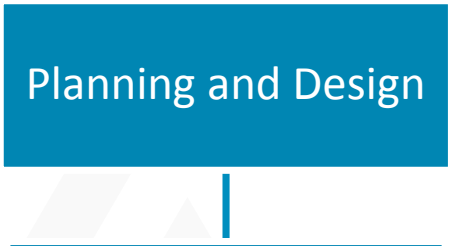

5.3: Modeling for Extreme Events

5.3.3: Simulating Cascades and N-k

5.3.4:

Interconnection Level Analysis
Multi-year Program Plan (MYPP)

- Project addresses computational scalability issues outlined in 5.3.3 


\section{Extreme Event Modeling 1.4.17 Project Team}

\section{Project Participants and Roles}

- Russell Bent (LANL): PI, Task Lead for 3.4: Most probable $\mathrm{N}-\mathrm{k}$ identification

- Yuri Makarov (PNNL): +1, Task Lead for 1.1: Integrating multiple temporal scales, 1.2: Inadequate Modeling-Integrating Protection System models

- Liang Min (LLNL): Task Lead for 1.3: Integrating renewables, 2.3: Parallel computing for massive dynamic contingency

- Feng Qiu (ANL): Task Lead for 2.1: Predicting critical cascading path

- Yaosuo Xue(ORNL): Task Lead for 2.2: Model Reduction Techniques

- Meng Yue (BNL): Task Lead for 3.1: Component Failure Probabilities

- Anthony Florita (NREL): Task Lead for 3.2: Mitigation Plan Modeling

- Jean-Paul Watson (SNL): Task Lead for 3.3: Worst Case N-k identification

\begin{tabular}{|l|l|l|l|}
\hline \multicolumn{4}{c}{ PROJECT FUNDING } \\
\hline Lab & FY16 \$ & FY17 \$ & FY18 \$ \\
\hline LANL & $155 \mathrm{~K}$ & $130 \mathrm{~K}$ & $145 \mathrm{~K}$ \\
\hline PNNL & $210 \mathrm{~K}$ & $235 \mathrm{~K}$ & $180 \mathrm{~K}$ \\
\hline LLNL & $160 \mathrm{~K}$ & $260 \mathrm{~K}$ & $210 \mathrm{~K}$ \\
\hline ANL & $125 \mathrm{~K}$ & $95 \mathrm{~K}$ & $125 \mathrm{~K}$ \\
\hline ORNL & $125 \mathrm{~K}$ & $95 \mathrm{~K}$ & $125 \mathrm{~K}$ \\
\hline BNL & $50 \mathrm{~K}$ & $45 \mathrm{~K}$ & $45 \mathrm{~K}$ \\
\hline NREL & $50 \mathrm{~K}$ & $45 \mathrm{~K}$ & $45 \mathrm{~K}$ \\
\hline SNL & $125 \mathrm{~K}$ & $95 \mathrm{~K}$ & $125 \mathrm{~K}$ \\
\hline
\end{tabular}

Industry and Academic Partners: GMLC, NERC, FERC, IEEE Cascading Failure Working Group, Dominion Virginia Power, PJM, ERCOT, UTK

- Webinar participation

- Power system data 


\section{Extreme Event Modeling 1.4.17 Approach}

- Cascade Modeling: Inadequate Modeling

$\square \quad$ Integrating multiple temporal scales

- Description: Develop new methods for modeling phenomena at different time multiple time scales

- Key Issues: Fundamentally different methods used at different time scales, difficult to integrate

- Novelty: Unique hybrid approach for combining phenomena and mathematics at different time scales

$\square \quad$ Integrating protection system models

- Description: Develop models of Zone 3 protection

- Key Issues: The extent and ordering of protection execution is often unknown

- Novelty: New methods for estimating the behavior of protection during cascades.

$\square \quad$ Integrating Renewables

- Description: Develop mathematical models and implementations of long-term wind dynamics

- Key Issues: No stability simulation platform that combines computational capabilities with models needed for assessing the implications of wind energy resources dynamics

- Novelty: new mathematical models of wind dynamics suitable for cascades

- Cascade Modeling: Computational Efficiency

$\square \quad$ Predicting critical cascading paths

- Description: Develop statistical methods for identifying cascading paths

- Key Issues: The number of possible cascade evolutions can be to large to enumerate

- Novelty: Models and software tools that statistically characterize component interactions that significantly limit the number cascade evolutions that need to be simulation

$\square \quad$ Model Reduction techniques

- Description: Methods and software for reducing the size of networks

- Key Issues: Network models can be too large for exhaustive cascade modeling

- Novelty: New approaches for model reduction based on measurement data $\square \quad$ Parallel computing for massive dynamic contingency analysis

- Description: Leverage HPC to improve efficiency of cascade modeling

- Key Issues: The number of cascades are too many to enumerate serially

- Novelty: Extensive leveraging of DOE and lab investments in HPC to improve computation by $500 x$

- Probabilistic N-k

$\square \quad$ Component failure probabilities

- Description: Develop probabilistic models of component failure based on data

- Key Issues: Utilities currently do not have rigorous approaches for build probabilistic models of failure

- Novelty: Formal probabilities for $\mathrm{N}-\mathrm{k}$

$\square \quad$ System failure probabilities

- Description: Develop probabilistic models of system failures based during extreme events

- Key Issues: Data is sparse for examples of extreme event system failures

- Novelty: Formal probabilistic of extreme event system failures

$\square \quad$ Worst-Case N-k Identification

- Description: Tools for identifying sets of $\mathrm{k}$ component failures with the biggest impact

- Key Issues: It is computationally intractable to find $k>3$ worst failures

- Novelty: New approaches for doubling the size of $k$

$\square \quad$ Most probable N-k Identification

- Description: Tools for identifying sets of $\mathrm{k}$ component failures whose probabilistic outcome is worst.

- Key Issues: Computationally very difficult to find sets of large $k$

- Novelty: Tools that combine probabilistic models with N-k optimization 


\section{Extreme Event Modeling 1.4.17 Approach}

- Cascade Modeling: Inadequate Modeling

Darallol comnuting for maccivo dvasmir continooncvanalysis

Integr Summary

- Core Question: What extreme events pose a risk and should be planned for?

- Cascade Modeling

$\square$ Integr - Goal: Realistic simulation of the outcomes of an extreme event

-

- Focus: Develop the realism, computation and tools to make this goal practical

$\bullet$

\begin{tabular}{c|l} 
Integr \\
$\bullet$
\end{tabular}$\stackrel{\text { N-k }}{\bullet}$ Goal: Identify extreme events of concern

- $\quad$ Focus: Develop the scale, computation, and tools to make this goal practical

- Historically: Goals pursued independently. Synergies at their intersection

- Example: Use $\mathrm{N}-\mathrm{k}$ to identify extreme events that require cascade modeling

- Example: Incorporate elements of cascade modeling into N-k

$\bullet$

-

- Approach: Push the science in these areas and make them practical for planning AND

$\bullet$ make the intersection between cascade modeling and $\mathrm{N}-\mathrm{k}$ a viable opportunity.

Mode

- Outcome: Open source software, demonstration on large-scale real systems,

- demonstrate the need for science at the intersection

- Novelty: New approaches for model reduction based on ind Design measurement data 


\section{Extreme Event Modeling 1.4.17 Accomplishments to Date}

\section{Cascade Modeling: Inadequate Modeling} Highlights

- Enhancement of Software: Dynamic Contingency Analysis Tools (DCAT) for cascading outage analysis (Year 2)

- Added integration with GE PSLF (Fall 2017)

- Developed new zone 3 protection models (Spring 2018)

- Incorporated corrective action models (Spring 2018)

- Discussed tech transfer

- GE PSLF

- Demonstration of analysis capabilities (Year 2)

- WECC planning model

- $\quad$ Stakeholder outreach (Year 2)

- Presentations to NERC, EPRI workshop, GE PSLF users group meeting and IEEE PES General Meeting

- Several WECC members are interested in testing DCAT

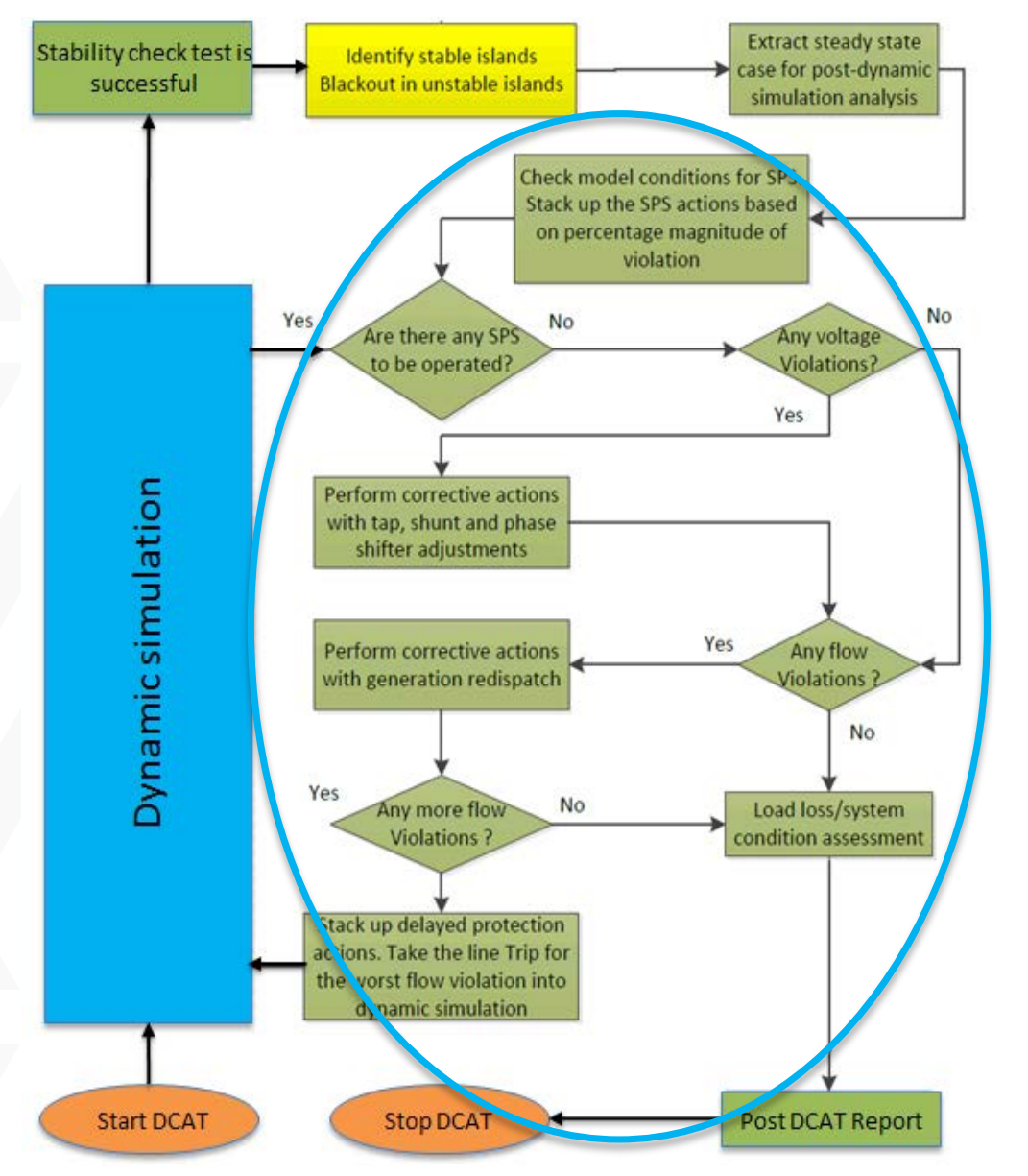




\section{Extreme Event Modeling 1.4.17}

\section{Accomplishments to date}

Cascade Modeling: Computation Highlights

- Leveraged parallelism of contingency event simulation (Year 2)

- $\quad$ Software Development: Contingency analysis that runs GE's Positive Sequence Load Flow (PSLF) in parallel on the Message Passing Interface (MPI) architecture (Year 2).

- Software Testing: $18.6 \mathrm{k}+$ single-element $(\mathrm{N}-\mathbf{1})$ contingencies were tested on WECC planning model (Spring 2018)

\section{Lessons learned:}

- Software compatibility-Porting PSLF from 32-bit Windows to 64-bit Linux environment and run on LLNL's Quartz HPC systems is a challenge.

- Parallelization efficiency measured with the longestrunning contingency.

\section{Market impact:}

- HPC-enabled contingency analysis with PSLF paves the way for analysis of extreme events in a (near-) real-time environment.

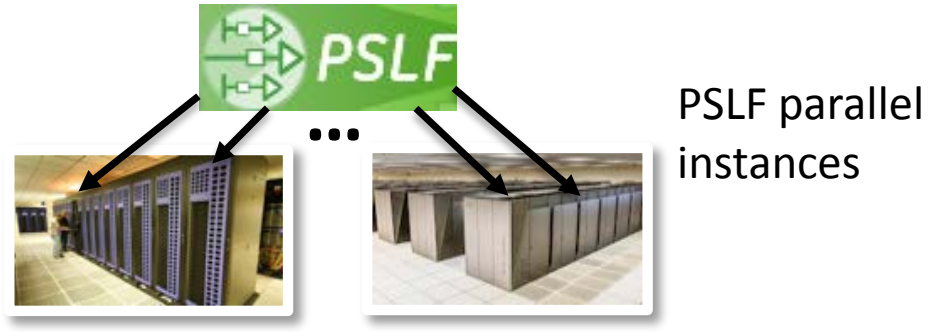

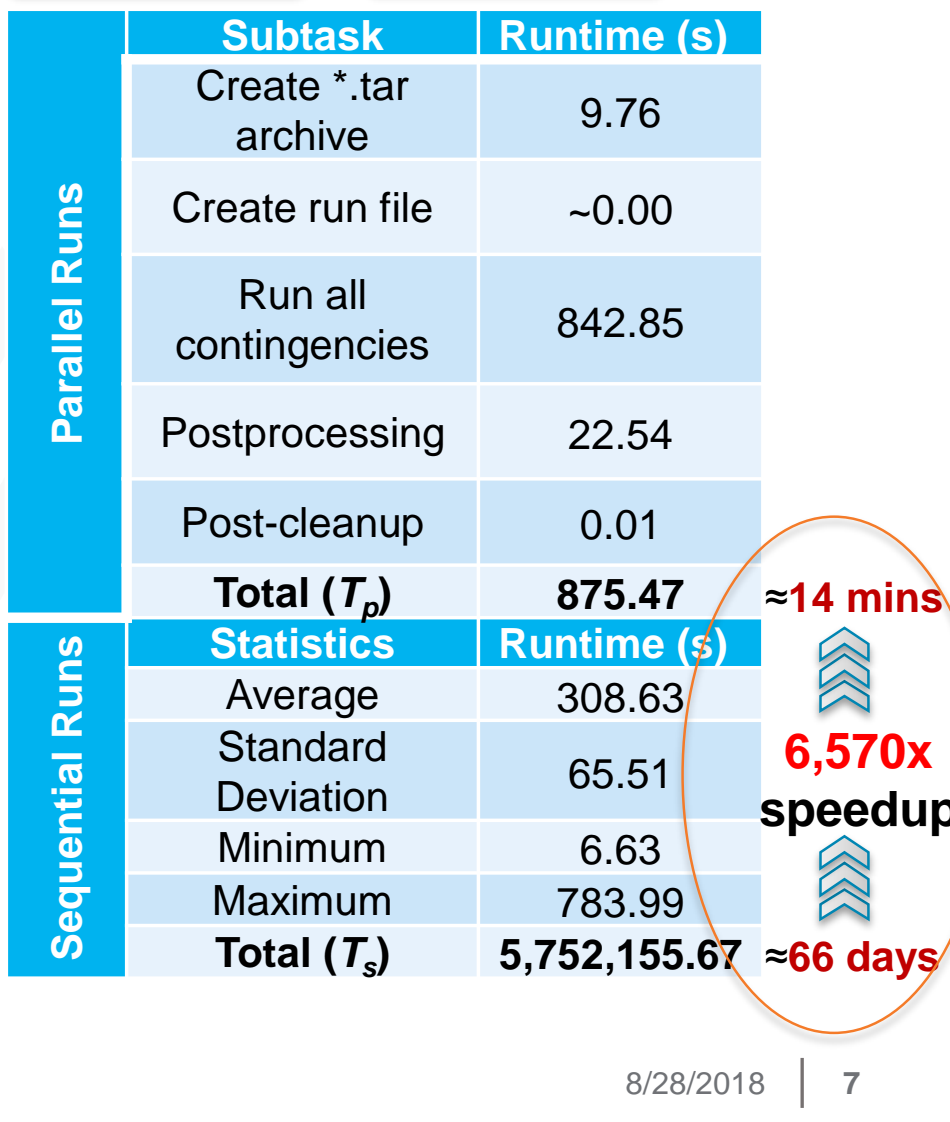




\section{Extreme Event Modeling 1.4.17 Accomplishments to Date}

N-k: Highlights

- $\quad$ Scaled N-k methods from systems with 100 's of nodes to 1000's of nodes

- Tested on AEP data sets

- > 10,000 nodes (Winter 2018)

- Developed N-k models based on models of the AC physics

- Higher fidelity then DC based modeling

- Developed novel N-k approach based on probabilistic failure models (Fall 2018)

\section{Lessons learned:}

- Probabilistic models identify different bad contingencies than deterministic models

- Deterministic $=$ worst case

- Probabilistic $\approx$ expected worst case

- Complimentary contingency lists

\section{Market impact:}

- Scalable N-k for near real time planning

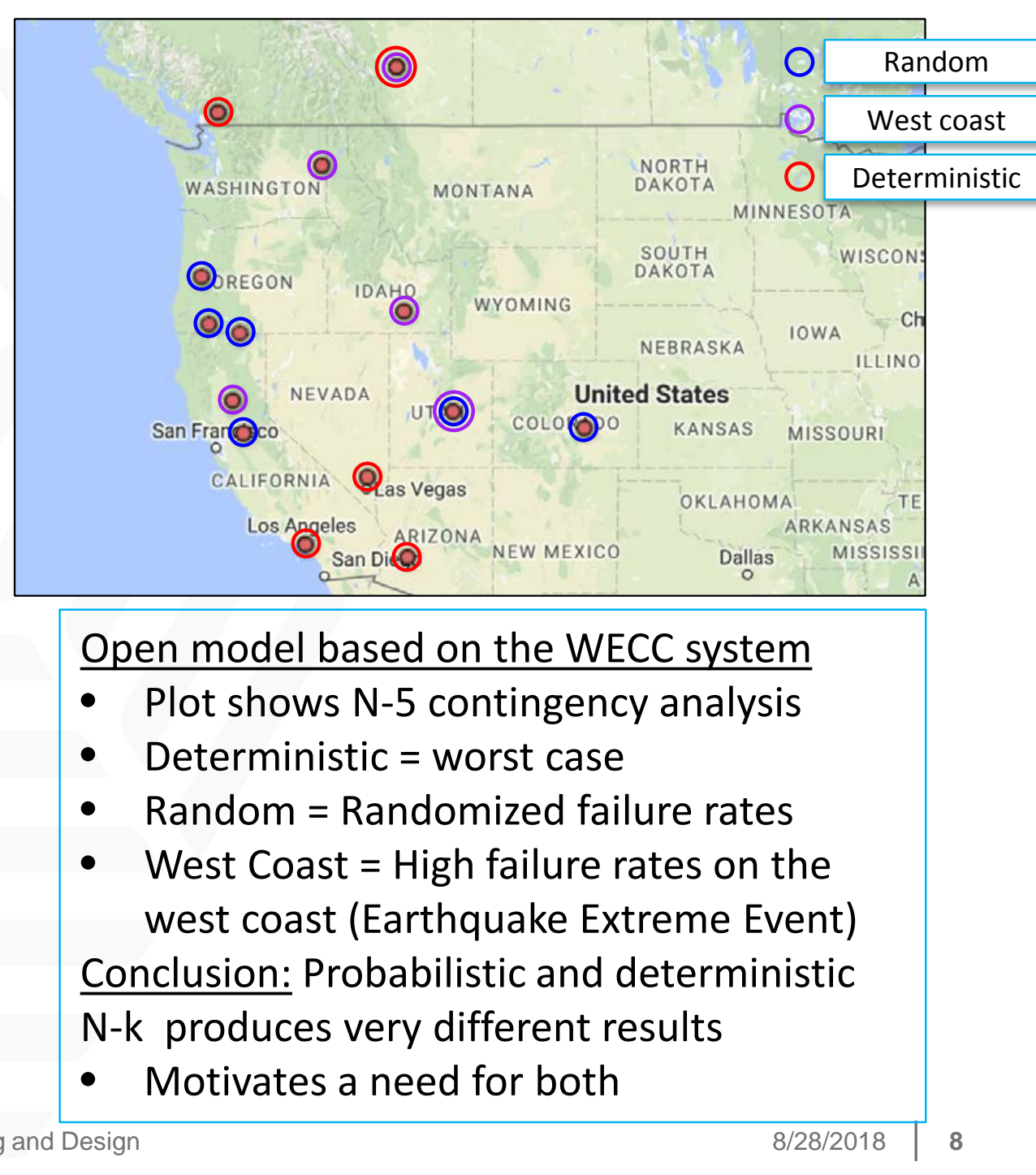




\section{Extreme Event Modeling 1.4.17 Accomplishments to Date}

Preliminary Cascade Modeling + N-k: Highlights

- $\quad \mathrm{N}-\mathrm{k}$ identification is based on steady state power flow equations

- Cascading analysis requires detailed transient studies to estimate impact

- Can N-k be used to select contingencies that require cascading analysis?

- A key open question and outcome of mid project meeting with NERC in Nov. 2017

\section{Approach}

- Rank N-k events using the N-k identification algorithm

- Perform cascading simulations on most severe N-k contingencies
The 20 most severe $\mathrm{N}-2$ contingencies identify high impact cascades

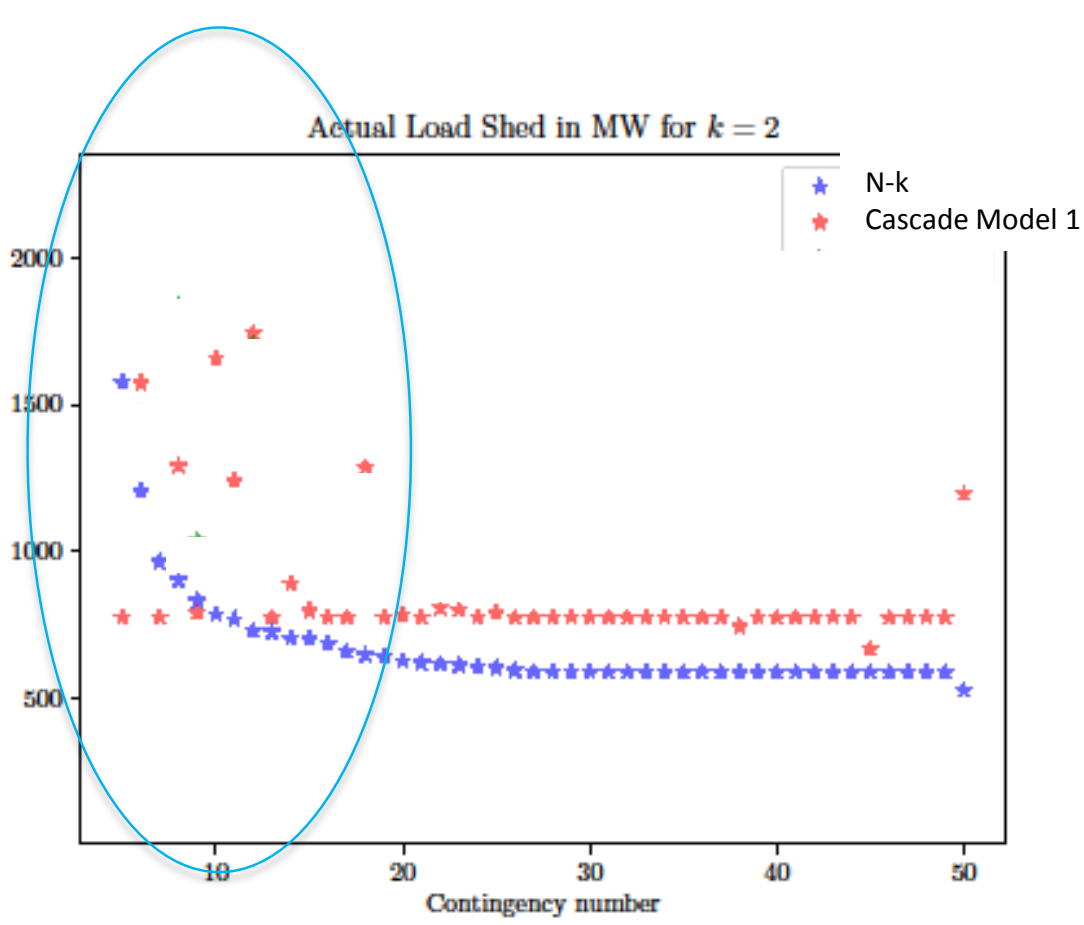

Blue stars: 50 most severe " $\mathrm{N}-2$ " contingencies from the $\mathrm{N}-\mathrm{k}$ analysis Red: cascading simulation 


\section{Extreme Event Modeling 1.4.17 Accomplishments to Date}

\section{Peer Reviewed Articles}

- $\quad$ E. Ciapessoni, D. Cirio, E. Cotilla-Sanchez, R. Diao, I. Dobson, A. Gaikwad, P. Henneaux, S. Miller, M. Papic, A. Pitto, J. Qi, N. Samaan, G. Sansavini, S. Uppalapati, and R. Yao, Benchmarking quasi-steady state cascading outage analysis methodologies, IEEE International Conference on Probabilistic Methods Applied to Power Systems (PMAPS), Boise, ID, USA, Jun. 2018. https://ieeexplore.ieee.org/abstract/document/8440212/

- $\quad$ X. Zhang, Y. Xue, S. You, and Y. Liu, U.S. Eastern Interconnection (EI) model reductions using a measurement-based approach, 2018 IEEE Pes T\&D Conference \& Exposition, Denver, CO, USA, April 17-19, 2018.

- $\quad$ X. Zhang, Y. Xue, Y. Liu, J. Chai, L. Zhu, and Y. Liu, Measurement-based System Dynamic, 2017 North American Power Symposium (NAPS), Morgantown, WV, Sept. 17-19, 2017. https://ieeexplore.ieee.org/document/8107178/

- $\quad$ Q. Huang, B. Vyakaranam, R. Diao, Y. Makarov, N. Samaan, M. Vallem, and E. Pajuelo, Modeling Zone-3 Protection with Generic Relay Models for Dynamic Contingency Analysis, PES General Meeting, 2017 https://ieeexplore.ieee.org/document/8274534/

- J. Qi, J. Wang, and K. Sun, Efficient Estimation of Component Interactions for Cascading Failure Analysis by EM Algorithm, IEEE Transactions on Power Systems, 33 (3): 3153-3161, 2018. https://ieeexplore.ieee.org/document/8070359/

- A. Florita, M. Folgueras, E. Wenger, V. Gevorgian, and K. Clark. Grid Frequency Extreme Event Analysis and Modeling in the Western Interconnections. Solar and Wind Integration Workshop, 2017. https://www.osti.gov/biblio/1407845

- Mallikarjuna Vallem, Bharat Vyakaranam, Jesse Holzer, Nader Samaan, Yuri V. Makarov, Ruisheng Diao, Qiuhua Huang, and Xinda Ke, Hybrid Cascading Outage Analysis of Extreme Events with Optimized Corrective Actions, 2017 Intelligent Systems Application to Power Systems (ISAP) Conference, San Antonio, September 2017. https://ieeexplore.ieee.org/document/8071375/

- $\quad$ K. Sundar, C. Coffrin, H. Nagarajan, R. Bent. Probabilistic N-k Failure-Identification for Power Systems, Networks, accepted for publication. https://onlinelibrary.wiley.com/doi/abs/10.1002/net.21806

- Mallikarjuna Vallem, Bharat Vyakaranam, Jesse Holzer, Nader Samaan, Yuri V. Makarov, Ruisheng Diao, Qiuhua Huang, and Xinda Ke, Hybrid Cascading Outage Analysis of Extreme Events with Optimized Corrective Actions, 2017 Intelligent Systems Application to Power Systems (ISAP) Conference, San Antonio, September 2017. https://ieeexplore.ieee.org/document/8071375/

- $\quad$ Bharat Vyakaranam, Nader Samaan, Mallikarjuna Vallem, Renke Huang, Ruisheng Diao, and Yuri Makarov, Brian Thomas, and William W Price. Modeling of Protection Relays using Generic Models in System Wide Power System Dynamic Simulation Studies. IEEE/PES General Meeting, Denver, August 2017.

- M. Korkali. Revealing the Role of Renewable Generation Models in Multiscale Power Grid Dynamic Simulation, Intelligent Power Grid of Tomorrow: Modeling, Planning, Control, and Operation, Reliable and Sustainable Electric Power and Energy Systems Management (Springer), under review. 


\section{Extreme Event Modeling 1.4.17 Next Steps and Future Plans}

\section{Project Next Steps (April 2019)}

- $\quad$ Open source software releases

- Demonstrations on a large scale system (WECC 2020 Planning Model)

- $\quad$ Preliminary results of value-added of N-k + cascading models

- $\quad$ Project continuation document

Future Plans and Follow on Activities (FY20++)

- Integrate extreme event analysis with mitigation

- $\quad$ Adjust operating points to reduce risk from extreme events

- Transition software to North American Resilience Model (NARM) Initiative efforts

- $\quad$ Automated recovery of non-converging cascade simulations

- Develop models of sources of extreme events and their impacts

- GMD, adversarial, natural gas stress

- Research on new risk analysis techniques 를를

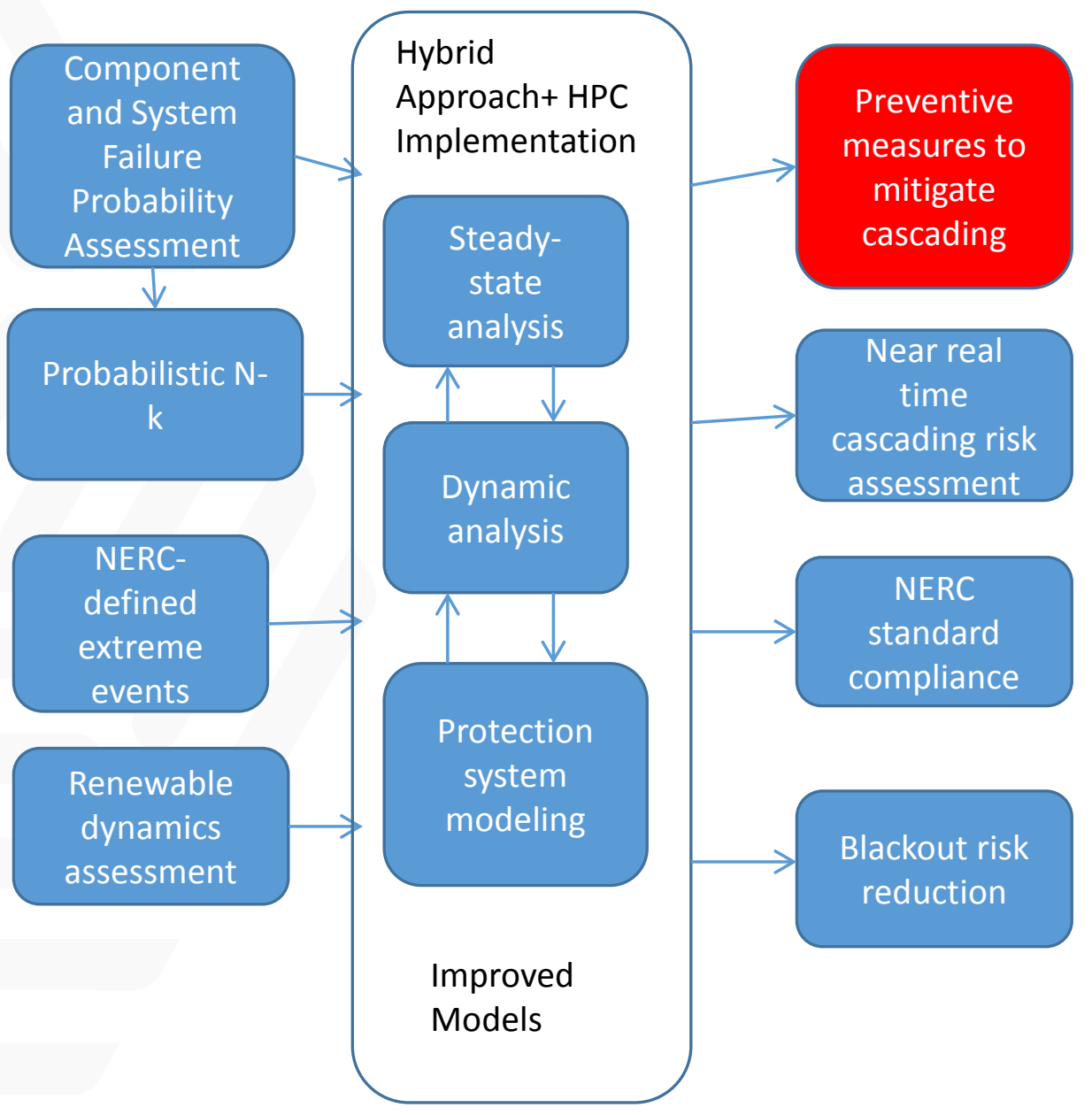

8/28/2018 


\section{Probabilistic N-k System Failures Accomplishments to date}

Light Spring Base Case Trip Path 66 (COI)

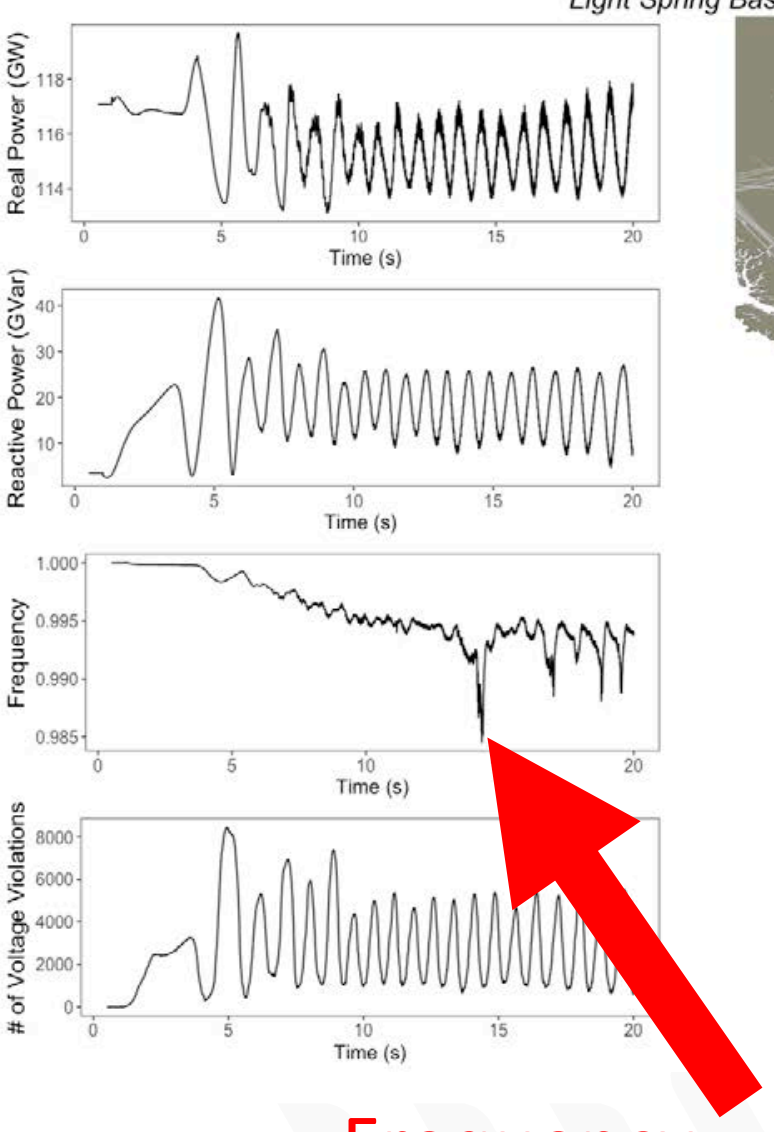

Frequency

Event

\section{- Overall Approach}

$\square$ Simulate faults (Generators, lines, and transformers) to develop of N-k probabilities

- WWSIS3 is used to model contingencies on scenarios:

$\square$ Scenario example: WECC model

- Transmission paths with $\leq 3$ lines-Trip all lines simultaneously

- Transmission paths with $>3$ lines-Trips subsets of 3 lines simultaneously

- Lessons Learned

$\square$ Studies of generator time series during contingency caused frequency events yield new understanding of coherency groups 


\section{Predicting Critical Cascading Paths}

\section{Accomplishments to date}

- Challenge: Lack of information about exact causes of outages

- Solution: Extract critical cascading path and failure propagation patterns

$\square$ EM algorithm solves an outage parameter estimation problem

- Outcomes: Leverage failure propagation patterns

$\square$ Learn failure propagation patterns from a small number of cascading simulations

$\square$ Approximate detailed cascading failure simulation with probabilistic interaction models simulation---more computationally efficient

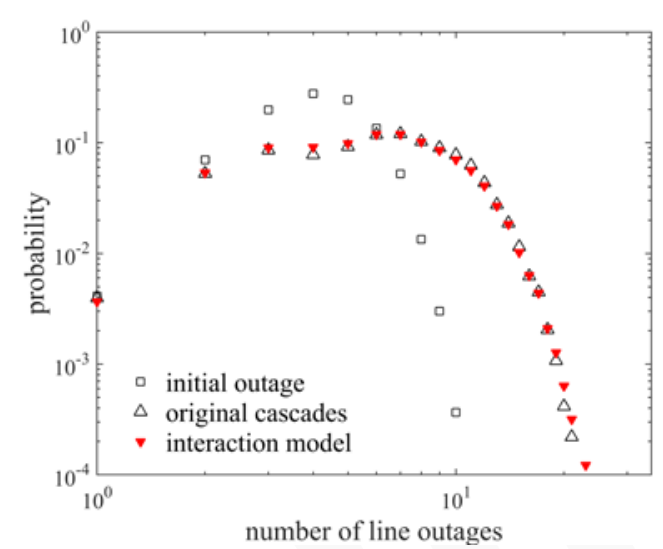

IEEE 118-bus system

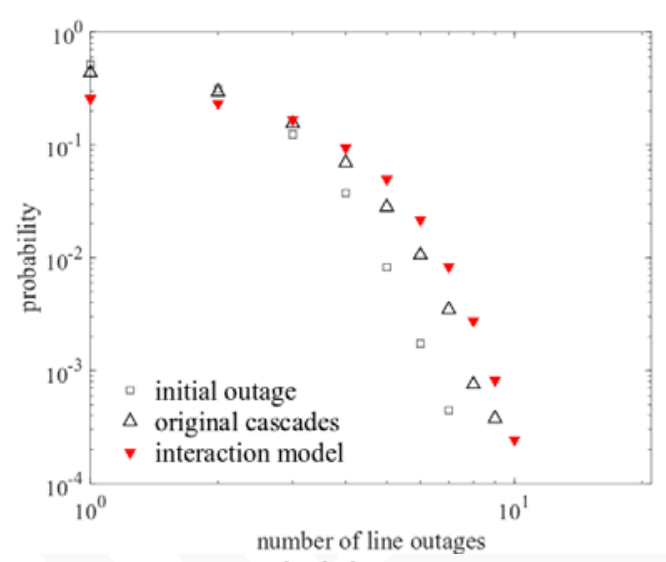

RTS-96 system

\begin{tabular}{|l|c|c|}
\hline & $\begin{array}{c}\text { IEEE 118- } \\
\text { Bus System }\end{array}$ & $\begin{array}{c}\text { RTS-96 } \\
\text { System }\end{array}$ \\
\hline $\begin{array}{l}\text { \# of cascades } \\
\text { needed }\end{array}$ & 41000 & 45000 \\
\hline $\begin{array}{l}\text { \# of cascades used } \\
\text { for estimation }\end{array}$ & 400 & 450 \\
\hline $\begin{array}{l}\text { Speed-up by } \\
\text { interaction model }\end{array}$ & 100.61 & 93.64 \\
\hline
\end{tabular}

J. Qi, J. Wang, and K. Sun, Efficient estimation of component interactions for cascading failure analysis by EM algorithm," IEEE Trans. Power Syst., vol. 33, no. 3, pp. 3153-3161, May 2018. https://ieeexplore.ieee.org/document/8070359/

F. Qiu and J. Qi,, Technical Report, Aug. 2018. Improving efficiency of cascading failure simulation for reliability test system by predicting critical u.s. pcascadjing path 


\section{Model Reduction}

Goal: Develop measurement-based dynamic model reductions Approach: Adapt model reductions to changing system states.

- Auto-regressive models with exogenous inputs (ARX)

- Transfer Functions

- $\quad$ Artificial Neural Networks (ANN)

- Best performer

Training Method: Simulate generation trips and line losses

Implementation: PSS/E and MATLAB/ANN co-simulation

Lessons Learned: Significantly reduced simulation time and good accuracy.

- Applied to the Eastern Interconnection network.
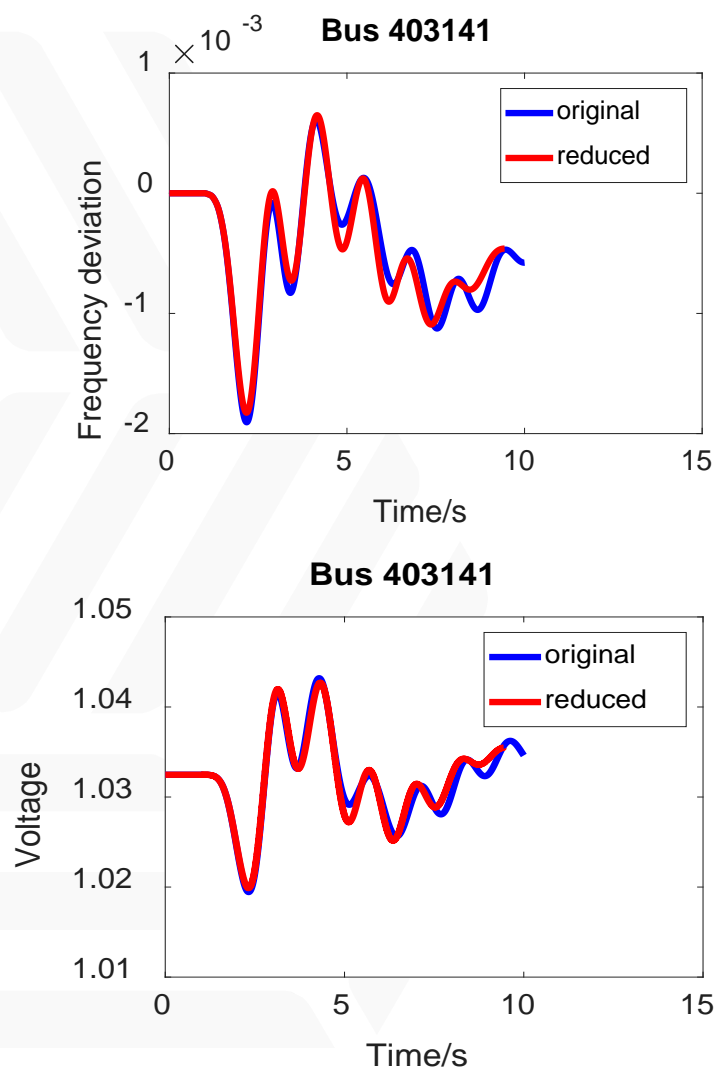

Reduced model a good predictor

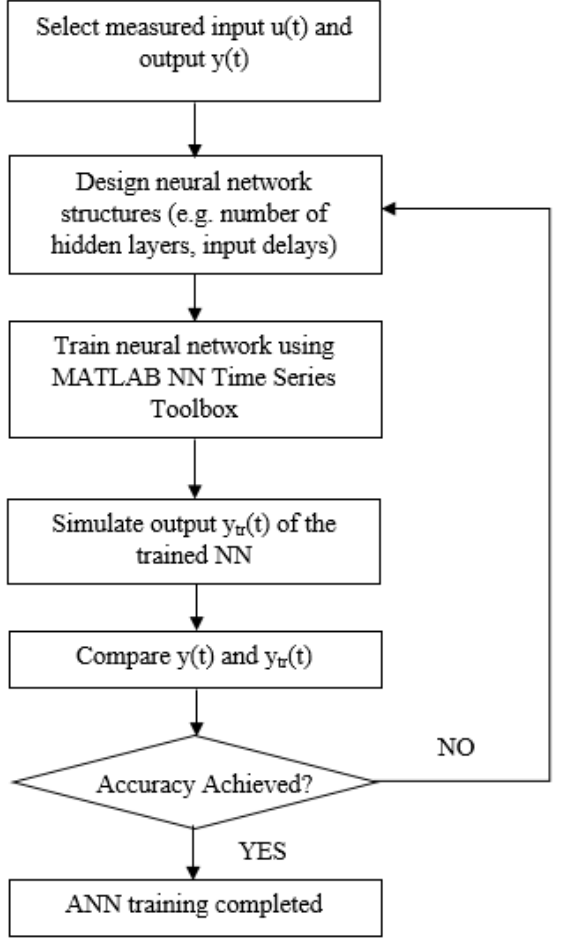

Algorithm Process Flow 


\section{Component Failure Probabilities Accomplishments to Date}

Accomplishment: Developed an enhanced reliability data repository

- Includes grid component outage data

- Includes renewable energy induced outages

Usage: Develop models of cascading failure based on a Markovian approach and an analytical quantification of system states

- Represents common modes and dependent outages as single outage events in a Markovian framework

- Model multiple outage modes of individual grid components

- Enables probabilistic risk assessment of cascading failures.

Presentation: IEEE Working Group on Probability Applications for Common Mode and dependent Events (PACME) during the PES General Meeting, August 7, 2018

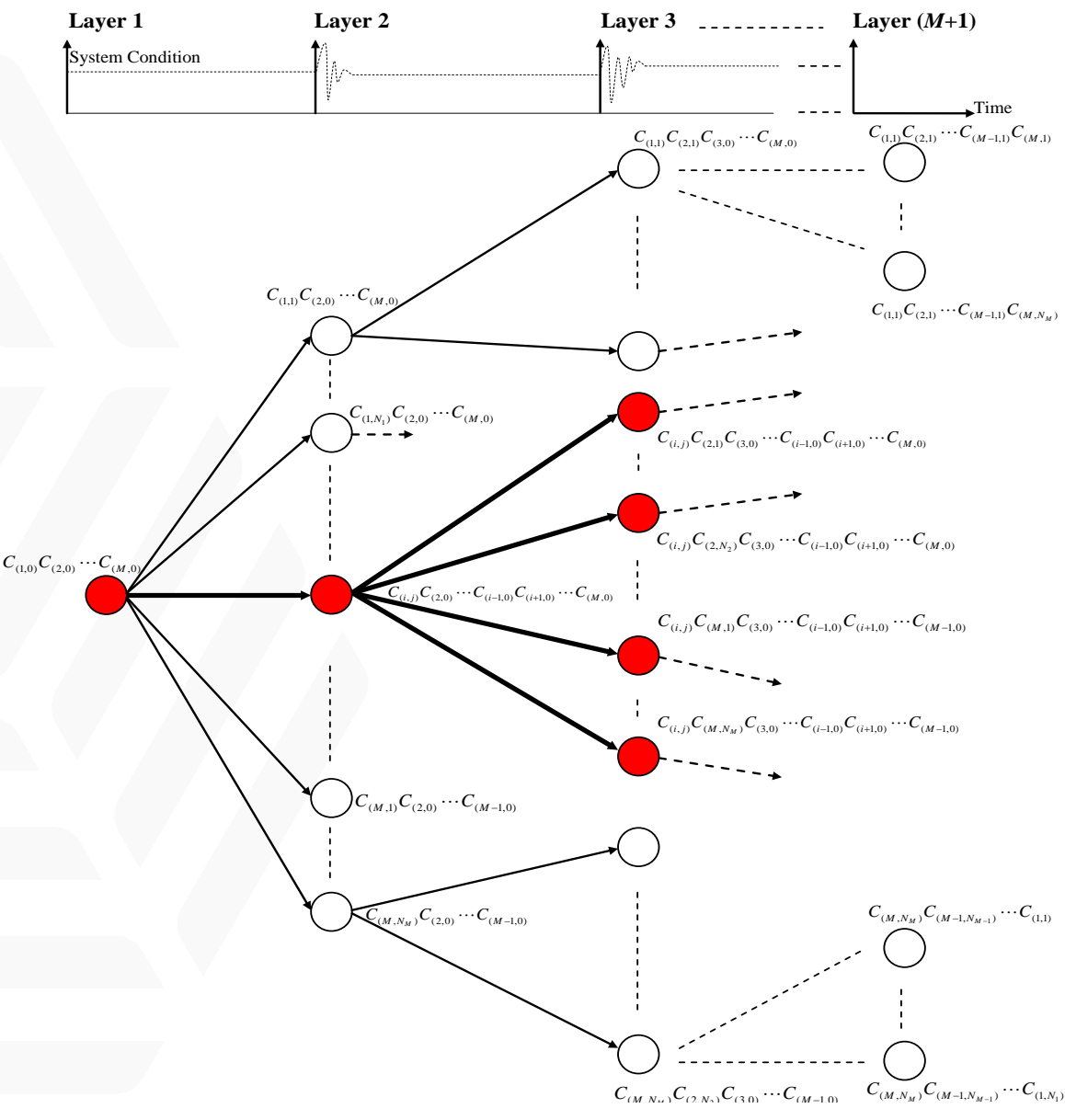

A Fully Expanded Markov Tree for cascading process 